\title{
Arteria coronaria única en paciente octogenario
}

\section{Single coronary artery in an octogenarian patient}

Luis Alfredo García Nielsen', Gerardo Rafael Padilla', Rafael Feldman', Juan Francisco Cuevas²

Palabras claves: coronaria única, anomalías congénitas Keywords: single coronary artery, coronary congenital anomaly

Revista Argentina de Cardioangiología Intervencionista 2019;10(3):125-126. https://doi.org/10.30567/RACI/201903/0125-0126

La arteria coronaria única (ACU) es una anomalía congénita con una incidencia de $0,026-0,06 \%{ }^{1}$. Se refiere al origen de la arteria coronaria izquierda o derecha a partir de un solo ostium en la raíz aórtica ${ }^{2}$. Aunque suele ser un hallazgo, puede producir angina, infarto de miocardio e incluso muerte súbi$\mathrm{ta}^{1}$. El mecanismo de la isquemia puede ser por angulaciones o por compresión cuando cruza entre los grandes vasos, generalmente relacionada con el esfuerzo. Cuando la coronaria derecha se origina desde la izquierda, son dos los trayectos posibles, el anterior y el interarterial, que implica mayor riesgo ${ }^{1}$. En una publicación reciente de nuestro grupo, en un paciente de 47 años, con taquicardia ventricular monomorfa sostenida y ACU, no tenía lesiones angiográficas ${ }^{1}$. También puede desarrollarse enfermedad aterosclerótica y los síntomas no deberse a la anomalía coronaria. Este caso se corresponde a un paciente de 81 años, sexo masculino, factores de riesgo hipertensión arterial y diabetes tipo 2; angioplastia previa de arteria circunfleja (CX) con stent metálico 12 años atrás. Actualmente refiere disnea. La perfusión miocárdica informó isquemia inferior. La coronariografía reveló una ACU con salida en el seno de Valsalva izquierdo, trayecto normal de descendente anterior (DA) al igual que la CX (Figura 1). Esta última continúa por el surco auriculoventricular con la coronaria derecha, que termina sin conexión con el seno correspondiente (Figura 2). Se observa además estenosis moderada en ostium de DA. El stent en CX permeable, con hiperplasia neointimal leve. La ACU, en ausencia de otra enfermedad congénita, no tiene implicancia pronóstica, y ha sido descrita en individuos de la octava década de la vida $^{3}$, como el ejemplo que presentamos. La actitud terapéutica se debe individualizar en función de los síntomas, la presencia de lesiones coronarias asociadas y la edad del paciente ${ }^{1}$.

\section{AGRADECIMIENTO}

Gustavo Centeno, Departamento de Enfermería, Hemodinamia, Sanatorio Sarmiento.

1. Servicio de Cardioangiología Intervencionista, Sanatorio Sarmiento, San Miguel de Tucumán.

2. Servicio de Cardiología, Sanatorio Sarmiento, San Miguel de Tucumán.

$\triangle$ Correspondencia: Dr. Luis A. García Nielsen, Servicio de Cardioangiología Intervencionista, Sanatorio Sarmiento, San Miguel de Tucumán. Igarcianielsen@yahoo.com.ar Los autores no declaran conflictos de intereses

Recibido: 22/05/2019| Aceptado: 28/06/2019

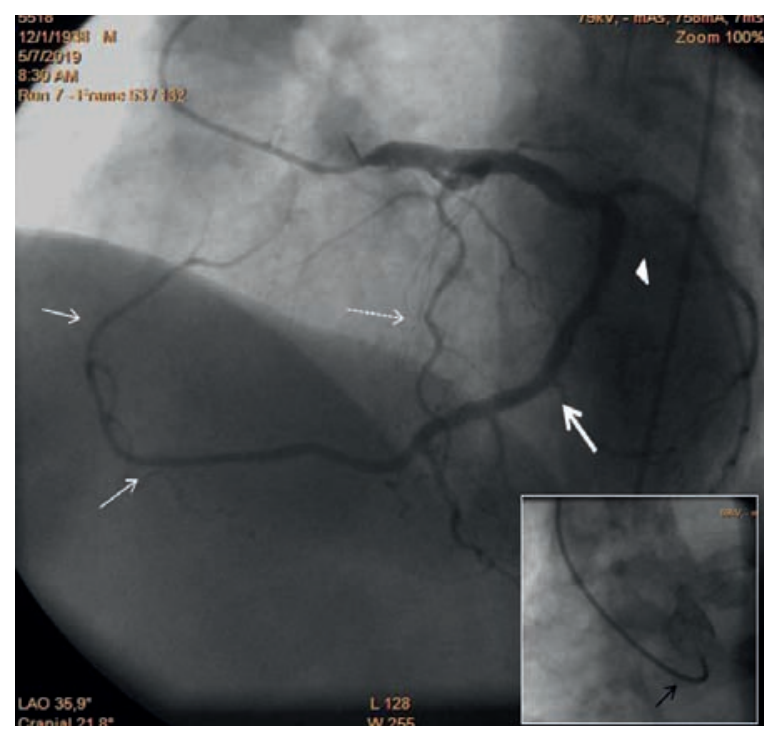

Figura 1. Coronariografía: proyección oblicua craneal izquierda que evidencia una arteria coronaria única con salida en el seno de Valsalva izquierdo, trayecto normal de descendente anterior (DA) (flecha punteada) al igual que la arteria circunfleja (CX, flecha gruesa). La flecha delgada señala la coronaria derecha (CD) en su recorrido por el surco AV y que se agota antes de desembocar en el seno de Valsalva derecho. Se observa además permeabilidad del stent en CX (cabeza de flecha). En el recuadro pequeño, la inyección de contraste en seno de Valsalva derecho demuestra ausencia de ostium de CD (flecha negra).

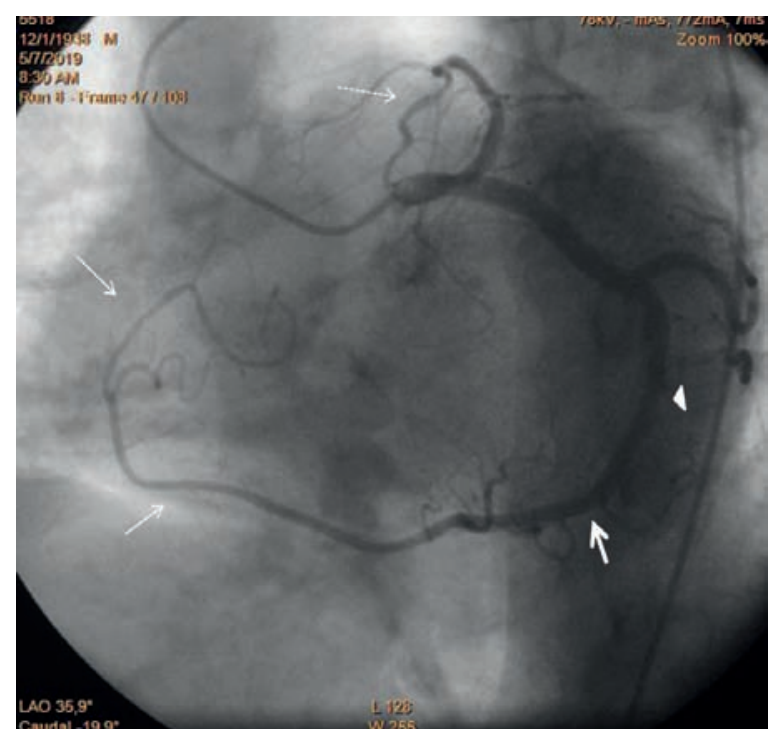

Figura 2. Coronariografía en proyección oblicua caudal izquierda. 


\section{BIBLIOGRAFÍA}

1. García-Nielsen L, Feldman R, Padilla G. Arteria coronaria única. Hallazgo ocasional en paciente con taquicardia ventricular. Rev Fed Arg Cardiol 2017;46(4).

2. Ozyurtlu F, Acet H, Zihni BM, Tasal A. A rare coronary artery anomaly: single coronary artery originate from right sinus Valsalva R-IIP sub-group type. Cardiol Res. 2012;3(3):140-2.
3. Morimoto H, Mukai S, Obata S, Hiraoka T. Incidental single coronary artery in an octogenarian with acute type A aortic dissection. Interact Cardiovasc Thorac Surg. 2012;15:307-8. 